\title{
Relieving oxidative stress in immune cells
}

Reticular dysgenesis (RD) is the most severe form of human severe combined immunodeficiency (SCID). It is associated with mutations in adenylate kinase 2 (AK2), the mitochondrial intermembrane space enzyme that regulates energy metabolism by reversibly catalyzing the reaction of ATP with AMP to generate two molecules of ADP. However, the impact of AK2 mutations on hematopoiesis has not been directly addressed. In this issue, Rissone et al. report that mitochondrial metabolic stress is an underlying cause of defective hematopoietic stem and progenitor cell (HSPC) maintenance and the failure of multilineage hematopoiesis in the absence of AK2.

$\mathrm{RD}$ is characterized by sensorineural hearing loss and the lack of innate and acquired immune responses. The lymphopenia and neutropenia in RD patients is caused by severe defects in lymphoid and myeloid differentiation. Hematopoietic stem cell transplantation (HSCT) is

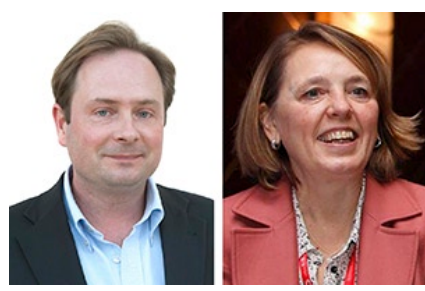

Insight from Kamil Kranc (left) and Elaine Dzierzak (right) currently the only therapeutic option, but RD patients often die from infections before HSCT can be performed. Thus, novel therapeutic strategies are required for this severe condition.

Rissone et al. modeled AK2 deficiency in zebrafish embryos and induced pluripotent stem cells (iPSCs) derived from RD patients. AK2-deficient zebrafish embryos displayed impaired survival of HSPCs and committed hematopoietic precursors, as well as arrests in lymphoid and myeloid development. Studies using RD patient-derived iPSCs harboring a homozygous AK2 mutation recapitulated myeloid maturation arrest (at the promyelocyte stage) observed in RD patient bone marrow and additionally showed defective erythropoiesis. AK2 deficiency resulted in ADP depletion, thus limiting the substrate availability for ATP production by the mitochondrial ATP synthase. This disturbance in cellular energy homeostasis was coupled with increased generation of reactive oxygen species (ROS). Notably, provision of ectopic antioxidants largely rescued AK2-deficient HSPCs and committed progenitors from apoptosis in the zebrafish embryo and restored lymphoid and myeloid differentiation in both zebrafish and iPSC models of RD.

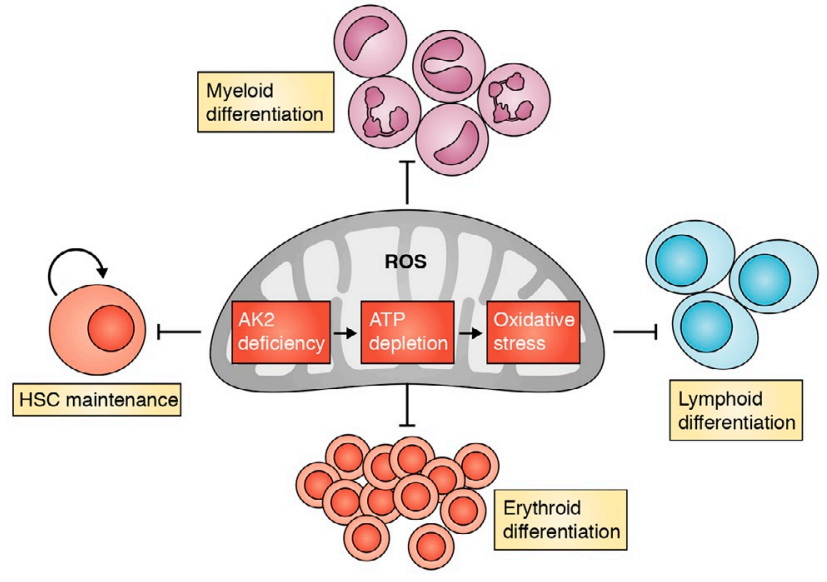

AK2 deficiency results in dysregulation of energy homeostasis and oxidative stress. Reactive oxygen species have a negative impact on HSC maintenance and multilineage differentiation.

By exploiting the strengths of these complementary experimental systems, the authors show that AK2 deficiency has broader deleterious consequences for hematopoietic differentiation than previously anticipated. Although antioxidants deplete ROS in AK2-deficient cells, they are unlikely to restore normal energy homeostasis. It is therefore somewhat surprising that antioxidant-treated cells lacking AK2 appear to undergo apparently normal energy-demanding differentiation. Nevertheless, this work elegantly illustrates that disease modeling in zebrafish together with studies on patient-derived iPSCs provides a powerful platform to reveal mechanisms underlying human immunodeficiency and identify novel targets for future therapeutic interventions. Although antioxidant treatment alone is unlikely to have long-term beneficial effects in RD, antioxidant administration may alleviate severe lymphopenia and neutropenia in RD patients and allow them to undergo curative HSCT.

Rissone,A., et al. 2015.J. Exp. Med. http://dx.doi.org/10.1084/jem.20141286

Kamil R. Kranc and Elaine Dzierzak, University of Edinburgh: Kamil.Kranc@ed.ac.uk and Elaine.Dzierzak@ed.ac.uk

\section{Saving $\boldsymbol{\beta}$ cell function in the NIK of time}

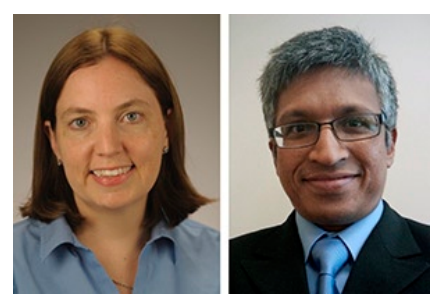

Insight from Kristin Tarbell (left) and Sushil Rane (right)
Type 2 diabetes is a disease of insulin resistance, and $\beta$ cell function is important for maintaining normal levels of insulin production. The canonical $N F-\kappa B$ signaling pathway has been well studied, but little is known about the noncanonical NF- $\mathrm{B}$ pathway in the context of pancreatic islet dysfunction. In this issue, Malle et al. demonstrate that the noncanonical NF- $\mathrm{B}-$-inducing kinase (NIK) is a negative regulator of $\beta$ cell function in diet-induced obesity.

In the canonical NF- $\kappa \mathrm{B}$ pathway, IKK activation leads to I $\kappa \mathrm{B} \alpha$ degradation and nuclear translocation of $\mathrm{p} 50$. In contrast, in the noncanonical NF- $\mathrm{B}$ pathway, $\mathrm{p} 100$ is processed into p52 that complexes with RelB for nuclear localization and transcription regulation. NIK activates $\mathrm{p} 100$ processing and is a central regulator of this noncanonical NF- $\mathrm{KB}$ pathway. 
The link between NIK and $\beta$ cell function was shown by Malle et al. using a wide variety of systems; drug-induced NIK activation resulted in impaired $\beta$ cell function in zebrafish, and elevated NIK levels in ex vivo cultures of rodent and human islets from diet-induced obese mice were associated with impaired glucosestimulated insulin secretory capacity. In addition, three complementary genetic approaches in mice showed that increased NIK activity (achieved via $\beta$ cellspecific knockout of components of the NIK degradation machinery) resulted in $\beta$ cell dysfunction.

The association of elevated NIK signaling and defective glucose-stimulated insulin secretion in the presence of high-fat diet is an important finding. Considering that elevated NIK also damages insulin-sensitive tissues such as muscle and liver, this study is consistent with NIK signaling as a key mechanism to propagate $\beta$ cell dysfunction and insulin resistance. TNF activates NIK and has been implicated as a driver of obesity-associated systemic inflammation that can lead to insulin resistance, primarily through an increase of inflammatory macrophages in the fat tissue. This study provides evidence that inflammation, and TNF specifically, may also be driving NIK activation in $\beta$ cells and that this could inhibit their ability to deal with the increased demand for insulin that occurs with insulin resistance.

The mechanistic details that underlie NIK-induced $\beta$ cell dysfunction are un-

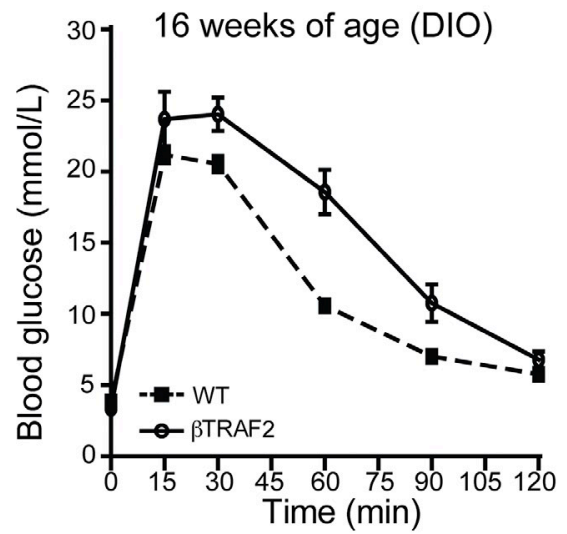

$\beta$ cell-specific TRAF2 knockout mice ( $\beta$ TRAF2 mice) have $\boldsymbol{\beta}$ cell-intrinsic NIK activation. In a diet-induced obesity model, $\beta$ TRAF2 mice demonstrate exacerbated glucose intolerance compared with wild-type mice. clear, and the relationship of this noncanonical loop of NF- $\mathrm{B}$ signaling with the canonical arm that has been already implicated in $\beta$ cell dysfunction and diabetes remains to be determined. Future studies should also address whether inhibition of NIK activity might be a promising therapeutic strategy for metabolic disease, by increasing $\beta$ cell function and attenuating hyperglycemia. Hyperglycemia causes complications of type 2 diabetes, and blocking inflammation-induced suppression of $\beta$ cell insulin production should improve glucose control and outcomes for patients with type 2 diabetes.

Acknowledgments: K. Tarbell and S. Rane are supported by the Division of Intramural Research, National Institute of Diabetes, Digestive and Kidney Diseases, National Institutes of Health.

Malle, E.K., et al. 2015.J. Exp. Med. http://dx.doi.org/10.1084/jem.20150218

Kristin V. Tarbell and Sushil G. Rane; National Institute of Diabetes and Digestive and Kidney Diseases, National Institutes of Health: tarbellK@niddk.nih.gov

\section{Immune-mediated viral clearance from the CNS without collateral damage}

The events that lead to the control of many infections are frequently associated with immunemediated collateral damage to surrounding cells and tissues. Nowhere is this more apparent than in the central nervous system (CNS), in part because of its immune privileged status. Lymphocytic choriomeningitis virus (LCMV) has provided a tractable model to study how antiviral effector responses, associated with chemokine production, lead to the recruitment of tissue-damaging myelomonocytes and fatal meningitis.

In this issue, Herz et al. report how "therapeutic T cells" derived from previously infected mice can clear LCMV from mice persistently infected from birth in the absence of collateral tissue damage. The authors found that the protective $\mathrm{T}$ cells did not express the chemokines typically associated with the recruitment of myelomonocytic cells that damage the blood-

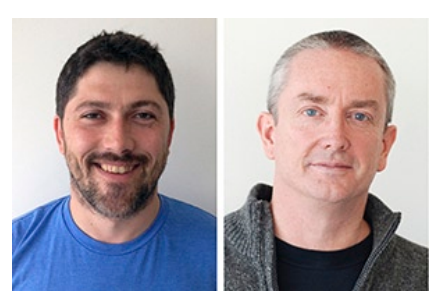

Insight from Christoph Konradt (left) and Christopher Hunter (right) brain barrier and cause tissue damage. The therapeutic $\mathrm{T}$ cells themselves were not cytolytic but rather produced IFN $\gamma$, which induced viral clearance in microglial cells and in neuronal cells. Because neurons are not considered responsive to IFN $\gamma$, it remains to be determined whether and how the noncytopathic bystander clearance of viral infection is mediated. It was notable that in persistently infected mice, microglia were a major reservoir of infection and the use of intravital imaging and transcriptional profiling revealed that the $\mathrm{T}$ cells interacted with microglial cells and prompted these CNS-resident macrophages to proliferate and present antigen. Thus, these findings indicate a central role for the therapeutic $\mathrm{T}$ cells in licensing of microglia to purge virus, present antigen, and presumably amplify the local antiviral $\mathrm{T}$ cell response. 


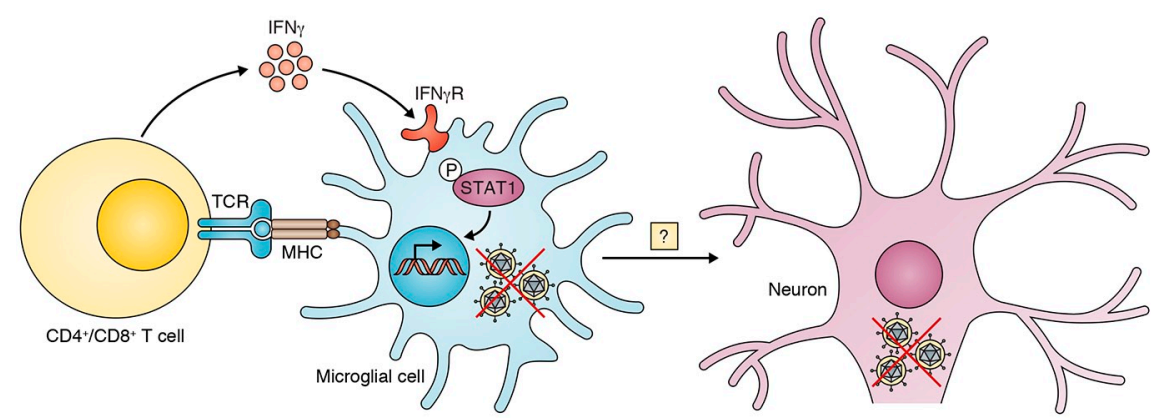

Therapeutic T cells produce IFN $\gamma$ and interact with microglial cells to activate STAT1 and mediate viral clearance and promote their ability to present antigen and to proliferate. Associated with these events is the noncytolytic clearance of virus from neurons by an unknown bystander mechanism.
A key challenge for neuroimmunologists is to understand how to promote effector responses that mediate local antimicrobial responses in the CNS with minimal recruitment of pathological bystander cells. A better understanding of the pathways that lead to the generation of the therapeutic $T$ cells used in this study and how they differ from those associated with pathology should provide clear insights into how to tailor a specific immune response to any given pathogen (or tumor) present in the CNS without causing immunopathology.

Herz, J., et al. 2015.J. Exp. Med. http://dx.doi.org/10.1084/jem.20142047

Christoph Konradt and Christopher A. Hunter, University of Pennsylvania: ckonradt@vet.upenn.edu and chunter@vet.upenn.edu

\section{Resolvins on the way to resolution}

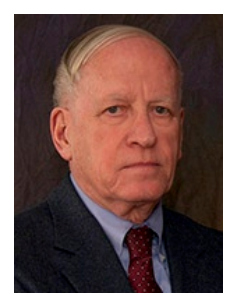

Insight from Peter Ward
Over the past several decades, lipid products have been found to exert both proinflammatory effects (e.g., prostaglandins and leukotrienes) and antiinflammatory activities (e.g., lipoxins and products derived from docosahexaenoic acid, including resolvins, protections, and moresins). Now, Chiang et al. describe a previously unrecognized role of GPR18 as a receptor for resolvin $(\mathrm{Rv})$ D2 that stimulates efferocytosis and mediates the resolution of inflammation.

GPR18, which is expressed on the surface of polymorphonuclear neutrophils (PMNs), monocytes, and macrophages, was identified as a receptor for RvD2 using unbiased GPCR- $\beta$-arrestin-based screening and functional sensing systems. Receptor-ligand interaction enhanced the innate immune functions (chemotaxis, transmigration, and phagocytosis) of these leukocytes and accelerated the resolution of various types of acute inflammation (caused either by infectious agents or after sterile ischemia-reperfusion injury). The infusion of RvD2 in infected mice impressively reduced the intensity of acute inflammatory responses (which often lead to tissue injury) and enhanced bacterial clearance. Experiments in GRP18-deficient mice confirmed the role of RvD2 in the resolution of inflammation.

Acute inflammatory responses, unless carefully regulated, can lead to substantial tissue damage that may become irreversible, resulting in fibrosis and loss of tissue or organ function. In some cases, for example in acute streptococcal pneumonia, an intense acute inflammatory response with a large buildup of PMNs, extensive edema, fibrin deposition, and hemorrhage resolves within 2-3 days, leaving behind normal lung tissue. The paper by Chiang et al. suggests that high levels of resolvins in the lung parenchyma have an important role to play, and the outcome of other infectious pneumonias may also depend on the appearance, or lack thereof, of resolvins and related lipids. However, unless tightly regulated, excessive activation of the innate immune responses stimulated by the RvD2-GRP18 axis could lead to tissue-damaging inflammatory responses. Perhaps there is a built-in mechanism (such as loss of cell surface receptors like GPR18) that automatically shuts these responses down? Further work will be required to determine whether it will be possible to target the resolvin system as a therapeutic intervention in human inflammatory disorders.

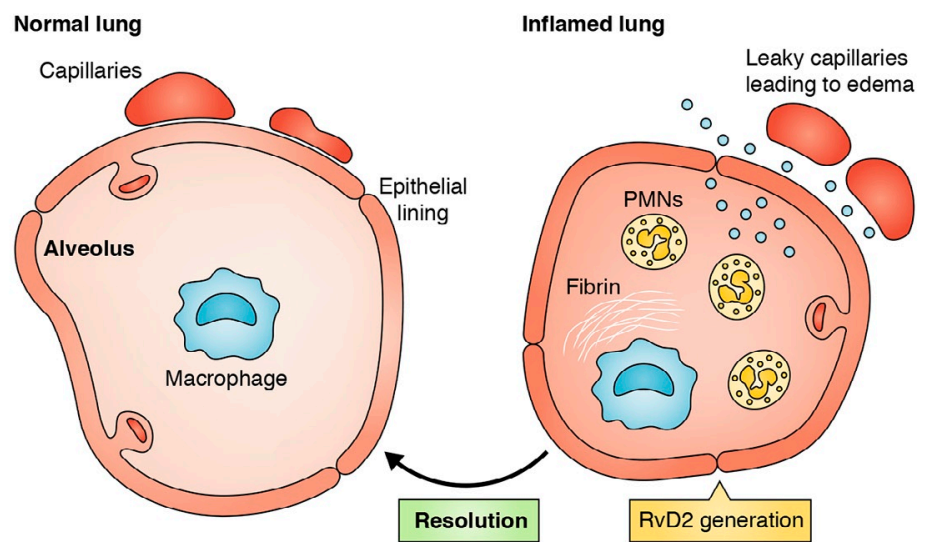

The presence of RvD2 enhances PMN recruitment into infected lungs, enhancing phagocytosis and chemotaxis of PMNs and phagocytosis (efferocytosis) of apoptotic PMNs. This results in the resolution of inflammation and the return of the lung to the preinjury state.

Chiang, N., et al. 2015.J. Exp. Med. http://dx.doi.org/10.1084/jem.20150225

Peter A. Ward, University of Michigan Medical School: pward@med.umich.edu 


\section{Self-targeting blocks malaria parasite invasion}

The development of drug resistance is a major problem in combating malaria caused by Plasmodium falciparum, the most deadly human malaria parasite. Resistance to artemisinin, the key component of current treatment regimens, is now being reported in parts of Asia. In this issue, Zenonos et al. report that it is possible to prevent parasite proliferation and clear a malaria infection using chimeric antibodies specific for a key host molecule required for parasite invasion of erythrocytes. Targeting a host protein rather than the parasite itself is much less likely to lead to the development of resistance, a problem that plagues treatments for virtually all infectious diseases.

In their study, Zenonos et al. generated recombinant chimeric antibodies with high affinity for the human erythrocyte surface receptor basigin. P. falciparum parasites bind to basigin as they invade erythrocytes, and this binding appears to be essential for parasite proliferation. The recombinant antibodies block this key interac-

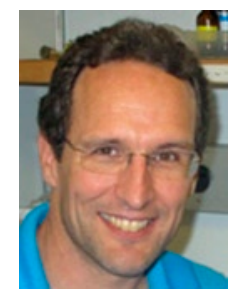

Insight from Kirk Deitsch tion, prevent erythrocyte invasion, and thus disrupt the replicative cycle that is crucial for the maintenance of an infection. Using a humanized mouse model, the authors showed that treatment with these antibodies leads to rapid clearance of an infection with no sign of recurrence.

The strategy of targeting host proteins raises obvious concerns about toxicity and side effects of the therapy. In the case of basigin, these concerns are somewhat alleviated by previous work using anti-basigin antibodies as therapies for cancer and graftversus-host disease, which were well tolerated. However, the authors reduced the likelihood of side effects by using chimeric antibodies that incorporated the human $\operatorname{IgG}_{1}$ and constant kappa chains, thus reducing the possibility of anti-mouse antibody responses. Further, they included an established set of mutations in the constant heavy chains that inhibit complement and Fc $\gamma-$

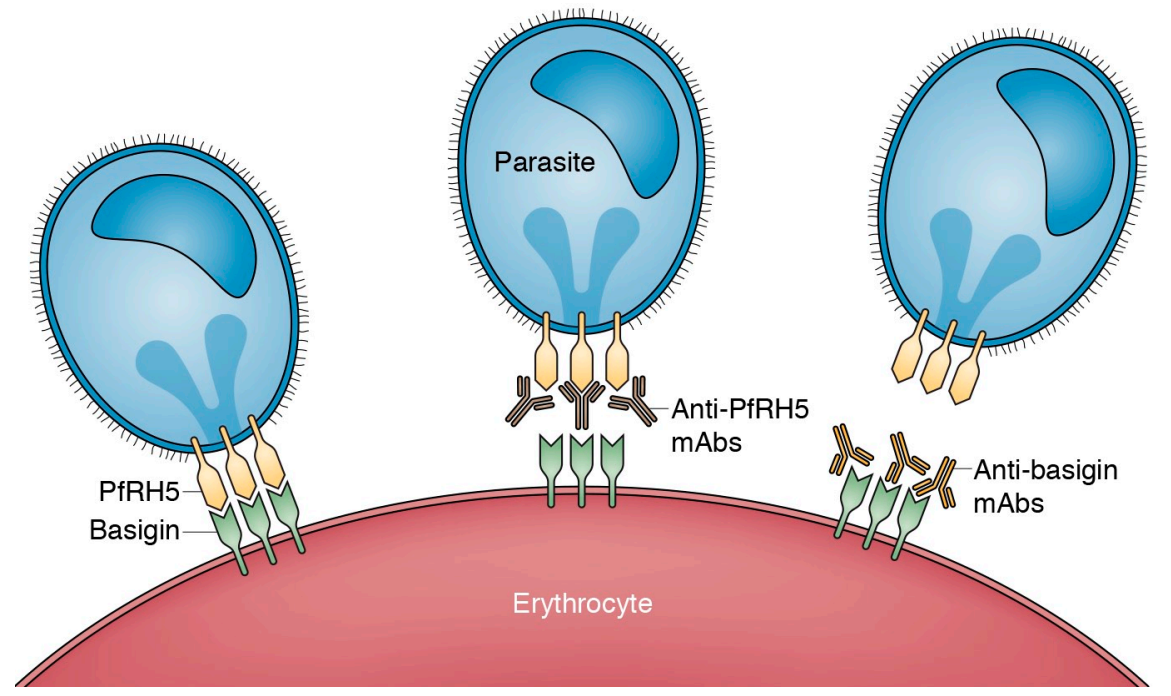

Invasion of host erythrocytes by $P$. falciparum malaria parasites and subsequent parasite proliferation both depend on binding of the host surface receptor basigin by the parasite ligand PfRH5 (left). Antibodies against PfRH5 can block invasion (middle), but this strategy is vulnerable to the development of mutations and polymorphisms in PfRH5. The alternative strategy, described by Zenonos et al., uses antibodies against basigin to block invasion (right). By targeting a host protein rather than the parasite, selection pressure for resistance is greatly reduced. receptor binding, thus significantly reducing the possibility of antibody effector functions targeted to the erythrocyte surface. The chimeric antibodies are therefore thought to disrupt parasite proliferation solely by blocking basiginparasite binding.

Malaria parasites, like most infectious organisms, have demonstrated a remarkable ability to develop resistance to widely used therapies. This raises the disturbing possibility that parasites resistant to all known therapies could develop in the near future, a predicament that is now confronting the tuberculosis community. By expanding our list of potential targets for disease intervention to several key host molecules-the first time this has been successfully demonstrated for malariawe can potentially create new therapies that are less vulnerable to the rapid generation of resistance.

Zenonos, Z.A., et al. 2015. J. Exp. Med. http://dx.doi.org/10.1084/jem.20150032

Kirk W. Deitsch, Weill Medical College of Cornell University: kwd2001@med.cornell.edu 